\title{
Some New Hermite-Hadamard Type Inequalities for Geometrically Convex Functions
}

\author{
İmdat İşcan \\ Department of Mathematics, Faculty of Arts and Sciences, Giresun University, 28100, Giresun, Turkey \\ *Corresponding Author: imdat.iscan@giresun.edu.tr
}

Copyright (c) 2013 Horizon Research Publishing All rights reserved.

\begin{abstract}
In this paper, some new integral inequalities of Hermite-Hadamard type related to the geometrically convex functions are established and some applications to special means of positive real numbers are also given.
\end{abstract}

Keywords Geometrically convex function, Hermite Hadamard type inequality

\section{Introduction}

This In this section, we firstly list several definitions and some known results.

Definition 1. Let $I$ be an interval in $\mathbb{R}$. Then $f: I \rightarrow \mathbb{R}$ is said to be convex function if

$$
f(t x+(1-t) y) \leq t f(x)+(1-t) f(y)
$$

for all $x, y \in I$ and $t \in[0,1]$.

Let $f: I \subseteq \mathbb{R} \rightarrow \mathbb{R}$ be a convex function defined on the interval $I$ of real numbers and $a, b \in I$ with $a<b$. The following double inequality is well known in the literature as Hermite-Hadamard integral inequality [1]

$$
f\left(\frac{a+b}{2}\right) \leq \frac{1}{b-a} \int_{a}^{b} f(x) d x \leq \frac{f(a)+f(b)}{2}
$$

For several recent results concerning Hadamard's inequality we refer the interested reader to [1-4, 6-8].

Definition 2 ( [5]). A function

$f: I \subset \mathbb{R}_{+}=(0, \infty) \rightarrow \mathbb{R}_{+}$is said to be GG-convex function (called in [8] geometrically convex function) if

$$
f\left(x^{t} y^{1-t}\right) \leq f(x)^{t} f(y)^{1-t}
$$

for $x, y \in I$ and $t \in[0,1]$.

In this paper, we will establish some new integral inequalities of Hermite-Hadamard-like type related to the geometrically convex functions and then apply these inequalities to special means.

\section{Main Results}

Theorem 1. Suppose that $f: I \subseteq \mathbb{R}_{+} \rightarrow \mathbb{R}_{+}$is geometrically convex and $a, b \in I$ with $a<b$. If $f \in L[a, b]$, then one has the inequalities :

$$
\begin{aligned}
& f(\sqrt{a b}) \leq \frac{1}{\ln b-\ln a} \int_{a}^{b} \frac{1}{x} \sqrt{f(x) f\left(\frac{a b}{x}\right)} d x \leq \frac{1}{\ln b-\ln a} \int_{a}^{b} \frac{f(x)}{x} d x \\
& \leq \frac{f(b)-f(a)}{\ln f(b)-\ln f(a)} \leq \frac{f(a)+f(b)}{2} .
\end{aligned}
$$

Proof. As $f$ is geometrically convex, we have, for all $x, y \in I$

$$
f(\sqrt{x y}) \leq \sqrt{f(x) f(y)} \leq \frac{f(x)+f(y)}{2} .
$$

Now, let $x=a^{1-t} b^{t}$ and $y=a^{t} b^{1-t}$ with $t \in[0,1]$. Then we get by (3) that:

$f(\sqrt{a b}) \leq \sqrt{f\left(a^{1-t} b^{t}\right) f\left(a^{t} b^{1-t}\right)} \leq \frac{f\left(a^{1-t} b^{t}\right)+f\left(a^{t} b^{1-t}\right)}{2}$ for all $t \in[0,1]$. Integrating this inequality on $[0,1]$, we deduce the inequalities (2).

Secondly, since $f$ is geometrically convex, we have, for all $t \in[0,1]$

$$
f\left(a^{t} b^{1-t}\right) \leq f(a)^{t} f(b)^{1-t} \leq t f(a)+(1-t) f(b) .
$$

Integrating this inequality on $[0,1]$, we get

$$
\frac{1}{\ln b-\ln a} \int_{a}^{b} \frac{f(x)}{x} d x \leq \frac{f(b)-f(a)}{\ln f(b)-\ln f(a)} \leq \frac{f(a)+f(b)}{2} .
$$

In order to prove next theorems, we need the following identity for differentiable functions. A consequence of the identities is that the author establishes some new inequalities connected with the inequalities (2) for the geometrically convex functions.

Lemma 1. Let $f: I \subseteq \mathbb{R}_{+} \rightarrow \mathbb{R}$ be a differentiable 
mapping on $I^{\circ}$, and $a, b \in I$, with $a<b$. If $f^{\prime} \in L[a, b]$, then

$$
\begin{aligned}
& f(\sqrt{a b})-\frac{1}{\ln b-\ln a} \int_{a}^{b} \frac{f(x)}{x} d x \\
& =\frac{(\ln b-\ln a)}{4}\left[\int_{0}^{1} t\left(\frac{b}{a}\right)^{\frac{t}{2}} f^{\prime}\left(a^{1-t}(a b)^{\frac{t}{2}}\right) d t\right. \\
& \left.-b \int_{0} t\left(\frac{a}{b}\right)^{\frac{t}{2}} f^{\prime}\left(b^{1-t}(a b) \frac{t}{2}\right) d t\right], \\
& \frac{f(a)+f(b)}{2}-\frac{1}{\ln b-\ln a} \int_{a}^{b} \frac{f(x)}{x} d x \\
& =\frac{(\ln b-\ln a)}{2}\left[\begin{array}{c}
1 \\
a \int t \\
0
\end{array}\left(\frac{b}{a}\right)^{t} f^{\prime}\left(a^{1-t} b^{t}\right) d t\right. \\
& \left.-b \int_{0}^{1} t\left(\frac{a}{b}\right)^{t} f^{\prime}\left(b^{1-t} a^{t}\right)\right] .
\end{aligned}
$$

Proof. Integrating by part and changing variables of integration yields

$$
\begin{aligned}
& \frac{(\ln b-\ln a)}{4}\left[\int_{0}^{1} t\left(\frac{b}{a}\right)^{\frac{t}{2}} f^{\prime}\left(a^{1-t}(a b)^{\frac{t}{2}}\right) d t\right. \\
&\left.-b \int_{0}^{1} t\left(\frac{a}{b}\right)^{\frac{t}{2}} f^{\prime}\left(b^{1-t}(a b)^{\frac{t}{2}}\right) d t\right] \\
&= \frac{1}{2}\left[\int_{0}^{1} t d f\left(a^{1-t}(a b)^{\frac{t}{2}}\right)+\int_{0}^{1} t d f\left(b^{1-t}(a b)^{\frac{t}{2}}\right)\right] \\
&= \frac{1}{2}\left[\left.t f\left(a^{1-t}(a b)^{\frac{t}{2}}\right)\right|_{0} ^{1}-\int_{0}^{1} f\left(a^{1-t}(a b)^{\frac{t}{2}}\right) d t\right] \\
&+ \frac{1}{2}\left[\left.t\left(b^{1-t}(a b)^{\frac{t}{2}}\right)\right|_{0} ^{1} \int_{0}^{1} f\left(b^{1-t}(a b)^{\frac{t}{2}}\right) d t\right] \\
&=f(\sqrt{a b})-\frac{1}{\ln b-\ln a} \int_{a} \frac{f(x)}{x} d x,
\end{aligned}
$$

and

$$
\begin{aligned}
& \frac{(\ln b-\ln a)}{2}\left[\int_{0}^{1} t\left(\frac{b}{a}\right)^{t} f^{\prime}\left(a^{1-t} b^{t}\right) d t d t\right. \\
& \left.-b \int_{0}^{1} t\left(\frac{a}{b}\right)^{t} f^{\prime}\left(b^{1-t} a^{t}\right) d t\right] \\
& =\frac{1}{2}\left[\int_{0}^{1} t d f\left(a^{1-t} b^{t}\right)+\int_{0}^{1} t d f\left(b^{1-t} a^{t}\right)\right] \\
& =\frac{1}{2}\left[\left.t f\left(a^{1-t} b^{t}\right)\right|_{0} ^{1}-\int_{0}^{1} f\left(a^{1-t} b^{t}\right) d t\right] \\
& +\frac{1}{2}\left[\left.t f\left(b^{1-t} a^{t}\right)\right|_{0} ^{1}-\int_{0}^{1} f\left(b^{1-t} a^{t}\right) d t\right] \\
& =\frac{f(a)+f(b)}{2}-\frac{1}{\ln b-\ln a} \frac{b}{a} \frac{f(x)}{x} d x .
\end{aligned}
$$

This completes the proof.

Theorem 2. Let $f: I \subseteq \mathbb{R}_{+} \rightarrow \mathbb{R}_{+}$be differentiable on $I^{\circ}$, and $a, b \in I^{\circ}$ with $a<b$ and $f^{\prime} \in L[a, b]$. If $\left|f^{\prime}\right|^{q}$ is geometrically convex on $[a, b]$ for $q \geq 1$, then

$$
\begin{aligned}
& \left|f(\sqrt{a b})-\frac{1}{\ln b-\ln a} \int_{a}^{b} \frac{f(x)}{x} d x\right| \leq \frac{\ln b-\ln a}{4}\left(\frac{1}{2}\right)^{1-\frac{1}{q}} \\
& \times\left\{a\left|f^{\prime}(a)\right|\left[g_{1}\left(\alpha\left(\frac{q}{2}\right)\right)\right]^{\frac{1}{q}}+b\left|f^{\prime}(b)\right|\left[g_{1}\left(\gamma\left(\frac{q}{2}\right)\right)\right]^{\frac{1}{q}}\right\} \\
& \left|\frac{f(a)+f(b)}{2}-\frac{1}{\ln b-\ln a} \int_{a}^{b} \frac{f(x)}{x} d x\right| \leq \frac{\ln b-\ln a}{2}\left(\frac{1}{2}\right)^{1-\frac{1}{q}} \\
& \times\left\{a\left|f^{\prime}(a)\right|\left[g_{1}(\alpha(q))\right] \frac{1}{q}+b\left|f^{\prime}(b)\right|\left[g_{1}(\gamma(q))\right] \frac{1}{q}\right\}
\end{aligned}
$$

Where

$$
g_{1}(\alpha)=\left\{\begin{array}{c}
\frac{1}{2}, \quad \alpha=1 \\
\frac{\alpha \ln \alpha-\alpha+1}{(\ln \alpha)^{2}}, \alpha \neq 1
\end{array}\right.
$$




$$
\begin{aligned}
& \alpha(u)=\left(\frac{b\left|f^{\prime}(b)\right|}{a\left|f^{\prime}(a)\right|}\right)^{u}, \\
& \gamma(u)=\left(\frac{a\left|f^{\prime}(a)\right|}{b\left|f^{\prime}(b)\right|}\right)^{u}, u>0 .
\end{aligned}
$$

Proof. (1) Since $\left|f^{\prime}\right|^{q}$ is geometrically convex on $[a, b]$, from lemma 1 and power mean inequality, we have

$$
\begin{aligned}
& \left|f(\sqrt{a b})-\frac{1}{\ln b-\ln a} \int_{a}^{b} \frac{f(x)}{x} d x\right| \\
& =\frac{(\ln b-\ln a)}{4}\left[\underset{0}{a} \int_{0}^{1} t\left(\frac{b}{a}\right)^{\frac{t}{2}}\left|f^{\prime}\left(a^{1-t}(a b) \frac{t}{2}\right)\right| d t\right. \\
& \left.+b \int_{0}^{1} t\left(\frac{a}{b}\right)^{\frac{t}{2}}\left|f^{\prime}\left(b^{1-t}(a b)^{\frac{t}{2}}\right)\right| d t\right] \\
& \leq \frac{a}{4} \ln \left(\frac{b}{a}\right)\left(\int_{0}^{1} t d t\right)^{1-\frac{1}{q}}\left(\int_{0}^{1} t\left(\frac{b}{a}\right)^{\frac{q t}{2}} \mid f^{\prime}\left(a^{\frac{2-t}{2}} b^{\frac{t}{2}}\right)^{q} d t\right)^{\frac{1}{q}} \\
& +\frac{b}{4} \ln \left(\frac{b}{a}\right)\left(\int_{0}^{1} t d t\right)^{1-\frac{1}{q}}\left(\int_{0}^{1} t\left(\frac{a}{b}\right)^{\frac{q t}{2}}\left|f^{\prime}\left(b^{\frac{2-t}{2}} a^{\frac{t}{2}}\right)\right|^{q} d t\right)^{\frac{1}{q}} \\
& \leq \frac{a}{4} \ln \left(\frac{b}{a}\right)\left(\frac{1}{2}\right)^{1-\frac{1}{q}} \\
& \times\left(\int_{0}^{1} t\left(\frac{b}{a}\right)^{\frac{q t}{2}}\left|f^{\prime}(b)\right|^{q(t / 2)}\left|f^{\prime}(a)\right|^{q((2-t) / 2)} d t\right)^{\frac{1}{q}} \\
& +\frac{b}{4} \ln \left(\frac{b}{a}\right)\left(\frac{1}{2}\right)^{1-\frac{1}{q}} \\
& \times\left(\int_{0}^{1} t\left(\frac{a}{b}\right)^{\frac{q t}{2}}\left|f^{\prime}(a)\right|^{q(t / 2)}\left|f^{\prime}(b)\right|^{q((2-t) / 2)} d t\right)^{\frac{1}{q}}
\end{aligned}
$$

$$
\begin{aligned}
& \leq \frac{\ln b-\ln a}{4}\left(\frac{1}{2}\right)^{1-\frac{1}{q}} \\
& \times\left\{a\left|f^{\prime}(a)\right|\left[g_{1}\left(\alpha\left(\frac{q}{2}\right)\right)\right]^{\frac{1}{q}}+b\left|f^{\prime}(b)\right|\left[g_{1}\left(\gamma\left(\frac{q}{2}\right)\right)\right]^{\frac{1}{q}}\right\} .
\end{aligned}
$$

(2) Since $\left|f^{\prime}\right|^{q}$ is geometrically convex on $[a, b]$, from lemma 1 and power mean inequality, we have we have

$$
\begin{aligned}
& \left|\frac{f(a)+f(b)}{2}-\frac{1}{\ln b-\ln a} \int_{a}^{b} \frac{f(x)}{x} d x\right| \\
& \leq \frac{(\ln b-\ln a)}{2}\left[\int_{0}^{1} t\left(\frac{b}{a}\right)^{t}\left|f^{\prime}\left(a^{1-t} b^{t}\right)\right| d t+b \int_{0}^{1} t\left(\frac{a}{b}\right)^{t}\left|f^{\prime}\left(b^{1-t} a^{t}\right)\right| d t\right] \\
& \leq \frac{a}{2} \ln \left(\frac{b}{a}\right)\left(\int_{0}^{1} t d t\right)^{1-\frac{1}{q}}\left(\int_{0}^{1} t\left(\frac{b}{a}\right)^{q t}\left|f^{\prime}\left(a^{1-t} b^{t}\right)\right|^{q} d t\right)^{\frac{1}{q}} \\
& +\frac{b}{2} \ln \left(\frac{b}{a}\right)\left(\int_{0}^{1} t d t\right)^{1-\frac{1}{q}}\left(\int_{0}^{1} t\left(\frac{a}{b}\right)^{q t}\left|f^{\prime}\left(b^{1-t} a^{t}\right)\right|^{q} d t\right)^{\frac{1}{q}} \\
& \leq \frac{a}{2} \ln \left(\frac{b}{a}\right)\left(\frac{1}{2}\right)^{1-\frac{1}{q}}\left(\int_{0}^{1} t\left(\frac{b}{a}\right)^{q t}\left|f^{\prime}(b)\right|^{q t}\left|f^{\prime}(a)\right|^{q(1-t)} d t\right)^{\frac{1}{q}} \\
& +\frac{b}{2} \ln \left(\frac{b}{a}\right)\left(\frac{1}{2}\right)^{1-\frac{1}{q}}\left(\int_{0}^{1} t\left(\frac{a}{b}\right)^{q t}\left|f^{\prime}(a)\right|^{q t}\left|f^{\prime}(b)\right|^{q(1-t)} d t\right)^{\frac{1}{q}} \\
& \leq \frac{\ln b-\ln a}{2}\left(\frac{1}{2}\right)^{1-\frac{1}{q}} \\
& \times\left\{a\left|f^{\prime}(a)\right|\left[g_{1}(\alpha(q))\right] \frac{1}{q}+b\left|f^{\prime}(b)\right|\left[g_{1}(\gamma(q))\right] \frac{1}{q}\right\} .
\end{aligned}
$$

This completes the proof.

If taking $q=1$ in Theorem 2, we can derive the following corollary.

Corollary 1. Let $f: I \subseteq \mathbb{R}_{+} \rightarrow \mathbb{R}_{+}$be differentiable on $I^{\circ}$, and $a, b \in I^{\circ}$ with $a<b$ and $f^{\prime} \in L[a, b]$. If $\left|f^{\prime}\right|$ is geometrically convex on $[a, b]$, then $[1]$

$$
\left|f(\sqrt{a b})-\frac{1}{\ln b-\ln a} \int_{a}^{b} \frac{f(x)}{x} d x\right|
$$




$$
\begin{gathered}
\leq \frac{\ln b-\ln a}{4}\left\{a\left|f^{\prime}(a)\right| g_{1}\left(\alpha\left(\frac{1}{2}\right)\right)+b\left|f^{\prime}(b)\right| g_{1}\left(\gamma\left(\frac{1}{2}\right)\right)\right\}, \\
\left|\frac{f(a)+f(b)}{2}-\frac{1}{\ln b-\ln a} \int_{a}^{b} \frac{f(x)}{x} d x\right| \\
\leq \frac{\ln b-\ln a}{2}\left\{a\left|f^{\prime}(a)\right| g_{1}(\alpha(1))+b\left|f^{\prime}(b)\right| g_{1}(\gamma(1))\right\} .
\end{gathered}
$$

Theorem 3. Let $f: I \subseteq \mathbb{R}_{+} \rightarrow \mathbb{R}_{+}$be differentiable on $I^{\circ}$, and $a, b \in I^{\circ}$ with $a<b$ and $f^{\prime} \in L[a, b]$. If $\left|f^{\prime}\right|^{q}$ is geometrically convex on $[a, b]$ for $q>1$, then

$$
\begin{aligned}
& \left|f(\sqrt{a b})-\frac{1}{\ln b-\ln a} \int_{a}^{b} \frac{f(x)}{x} d x\right| \\
& \leq \frac{\ln b-\ln a}{4}\left(\frac{q-1}{2 q-1}\right)^{1-\frac{1}{q}} \\
& \times\left\{a\left|f^{\prime}(a)\right|\left[g_{2}\left(\alpha\left(\frac{q}{2}\right)\right)\right]^{\frac{1}{q}}+b\left|f^{\prime}(b)\right|\left[g_{2}\left(\gamma\left(\frac{q}{2}\right)\right)\right]^{\frac{1}{q}}\right\}, \\
& \left|\frac{f(a)+f(b)}{2}-\frac{1}{\ln b-\ln a} \int_{a}^{b} \frac{f(x)}{x} d x\right| \\
& \leq \frac{\ln b-\ln a}{2}\left(\frac{q-1}{2 q-1}\right)^{1-\frac{1}{q}} \\
& \times\left\{a\left|f^{\prime}(a)\right|\left[g_{2}(\alpha(q))\right]^{\frac{1}{q}}+b\left|f^{\prime}(b)\right|\left[g_{2}(\gamma(q))\right]^{\frac{1}{q}}\right\}
\end{aligned}
$$

where inequality [1]

$$
g_{2}(\alpha)=\left\{\begin{array}{c}
1, \quad \alpha=1 \\
\frac{\alpha-1}{\ln \alpha}, \alpha \neq 1
\end{array},\right.
$$

and $\alpha(u), \gamma(u)$ are the same as in (7).

Proof. (1) Since $\left|f^{\prime}\right|^{q}$ is geometrically convex on $[a, b]$, from lemma 1 and Hölder inequality, we have then

$$
\begin{aligned}
& \left|f(\sqrt{a b})-\frac{1}{\ln b-\ln a} \int_{a}^{b} \frac{f(x)}{x} d x\right| \\
\leq & \frac{\ln \left(\frac{b}{a}\right)}{4}\left|\int_{0}^{a} t\left(\frac{b}{a}\right)^{\frac{t}{2}}\right| f^{\prime}\left(a^{1-t}(a b) \frac{t}{2}\right) \mid d t
\end{aligned}
$$

$$
\begin{aligned}
& \left.+b \int_{0}^{1} t\left(\frac{a}{b}\right)^{\frac{t}{2}}\left|f^{\prime}\left(b^{1-t}(a b)^{\frac{t}{2}}\right)\right| d t\right] \\
& \leq \frac{\ln \left(\frac{b}{a}\right)}{4}\left|\int_{0}^{1} t\left(\frac{b}{a}\right)^{\frac{t}{2}}\right| f^{\prime}\left(a^{1-t}(a b) \frac{t}{2}\right) \mid d t \\
& \left.+b \int_{0}^{1} t\left(\frac{a}{b}\right)^{\frac{t}{2}}\left|f^{\prime}\left(b^{1-t}(a b)^{\frac{t}{2}}\right)\right| d t\right] \\
& \leq \frac{a}{4} \ln \left(\frac{b}{a}\right)\left(\int_{0}^{1} t^{\frac{q}{q-1}} d t\right)^{1-\frac{1}{q}}\left(\int_{0}^{1}\left(\frac{b}{a}\right)^{\frac{q t}{2}} \mid f^{\prime}\left(a^{1-t}(a b)^{\frac{t}{2}}\right)^{q} d t\right)^{\frac{1}{q}} \\
& +\frac{b}{4} \ln \left(\frac{b}{a}\right)\left(\int_{0}^{1} t^{\frac{q}{q-1}} d t\right)^{1-\frac{1}{q}}\left(\int_{0}^{1}\left(\frac{a}{b}\right)^{\frac{q t}{2}}\left|f^{\prime}\left(b^{1-t}(a b)^{\frac{t}{2}}\right)\right|^{q} d t\right)^{\frac{1}{q}} \\
& \leq \frac{a}{4} \ln \left(\frac{b}{a}\right)\left(\frac{q-1}{2 q-1}\right)^{1-\frac{1}{q}}\left(\int_{0}^{1}\left(\frac{b}{a}\right)^{\frac{q t}{2}}\left|f^{\prime}(b)\right|^{\frac{q t}{2}}\left|f^{\prime}(a)\right|^{q((2-t) / 2)} d t\right)^{\frac{1}{q}} \\
& +\frac{b}{4} \ln \left(\frac{b}{a}\right)\left(\frac{q-1}{2 q-1}\right)^{1-\frac{1}{q}}\left(\int_{0}^{1}\left(\frac{a}{b}\right)^{\frac{q t}{2}}\left|f^{\prime}(a)\right|^{\frac{q t}{2}}\left|f^{\prime}(b)\right|^{q((2-t) / 2)} d t\right)^{\frac{1}{q}} \\
& \leq \frac{\ln b-\ln a}{4}\left(\frac{q-1}{2 q-1}\right)^{1-\frac{1}{q}} \\
& \times\left\{a\left|f^{\prime}(a)\right|\left[g_{2}\left(\alpha\left(\frac{q}{2}\right)\right)\right]^{\frac{1}{q}}+b\left|f^{\prime}(b)\right|\left[g_{2}\left(\gamma\left(\frac{q}{2}\right)\right)\right]^{\frac{1}{q}}\right\} \text {. }
\end{aligned}
$$

\section{Application to Special Means}

Let us recall the following special means of two nonnegative number $a, b$ with $b>a$ :

1. The arithmetic mean

$$
A=A(a, b):=\frac{a+b}{2} .
$$

2. The Geometric mean

$$
G=G(a, b):=\sqrt{a b} .
$$

3. The Logarithmic mean 


$$
L=L(a, b):=\frac{b-a}{\ln b-\ln a} .
$$

4. The p-Logarithmic mean

$$
L_{p}=L_{p}(a, b):=\left(\frac{b^{p+1}-a^{p+1}}{(p+1)(b-a)}\right)^{\frac{1}{p}}, p \in \mathbb{R} \backslash\{-1,0\} .
$$

Proposition 1. Let $0<a<b \leq 1$ and $q \geq 1$. Then for $n>1$

$$
\begin{aligned}
& \left|G\left(a^{n+1}, b^{n+1}\right)-L_{n}^{n}(a, b) L(a, b)\right| \leq\left(\frac{(b-a)(n+1) q}{4 L(a, b)}\right)^{1-\frac{1}{q}} \frac{1}{2 q} \\
& \times\left\{a^{\frac{n+1}{2}}\left[b^{\frac{(n+1) q}{2}}-\frac{\frac{(n+1) q}{2}-1}{\frac{(n+1) q}{2}-1}(a, b) L(a, b)\right]^{\frac{1}{q}}\right. \\
& \left.+b \frac{n+1}{2}\left[\frac{\frac{(n+1) q}{2}-1}{\frac{(n+1) q}{2}-1}(a, b) L(a, b)-a \frac{(n+1) q}{2}\right]^{\frac{1}{q}}\right\} \\
& \left|A\left(a^{n+1}, b^{n+1}\right)-L_{n}^{n}(a, b) L(a, b)\right| \leq\left(\frac{(b-a)(n+1) q}{2 L(a, b)}\right)^{1-\frac{1}{q}} \frac{1}{2 q} \\
& \times\left\{\left[b^{(n+1) q}-L_{(n+1) q-1}^{(n+1) q-1}(a, b) L(a, b)\right]^{\frac{1}{q}}\right. \\
& \left.+\left[L_{(n+1) q-1}^{(n+1) q-1}(a, b) L(a, b)-a^{(n+1) q}\right]^{\frac{1}{q}}\right\} .
\end{aligned}
$$

\section{REFERENCES}

[1] S.S. Dragomir and C.E.M. Pearce, Selected topics on Hermite-Hadamard type inequalities and applications, RGMIA Monographs, 2000. Online available from http://rgmia.vu.edu.au/monographs/hermite hadamard.html.

[2] I. Iscan, A new generalization of some integral inequalities and their applications, International Journal of Engineering and Applied sciences, Vol. 3 (3) (2013), 17-27.

[3] I. Iscan, A new generalization of some integral inequalities for $(\alpha, m)$-convex functions, Mathematical Sciences, Vol. 7 (22) (2013). doi:10.1186/2251-7456-7-22.

[4] U.S. Kirmaci, Inequalities for differentiable mappings and applications to special means of real numbers and to midpoint formula, Appl. Math. Comput., Vol. 147 (2004), 91-95.

[5] C. P. Niculescu, Convexity according to the geometric mean, Math. Inequal. Appl. 3 (2) (2000), 155-167. Online available from http://dx.doi.org/10.7153/mia-03-19

[6] M. Z. Sarikaya, E. Set, and M. E.Ozdemir, On new inequalities of Simpson's type for convex functions, RGMIA: Research Report Collection, Vol. 13 (2) (2010), article 2.

[7] B.-Y. Xi, R.-F. Bai, F. Qi, Hermite-Hadamard type inequalities for the $\mathrm{m}$ - and $(\alpha, m)$-geometrically convex functions, Aequat. Math. Vol. 84 (2012), 261-269.

[8] T.-Y. Zhang, A.-P. Ji and F. Qi, On Integral Inequalities of Hermite-Hadamard Type for s-Geometrically Convex 
Functions, Abstract and Applied Analysis, Vol. 2012 (2012), Article ID 560586, 14 pages, doi:10.1155/2012/560586. 